\title{
Electron-Photon Interactions: Time and Energy Relationships
}

\author{
Tibor Berceli*
}

Budapest University of Technology and Economics, Hungary

\begin{abstract}
The paper presents a simple, new approach providing results on some properties of electron-photon interactions like their processing time, energy density, optical pulse length. The main goal of the investigation is to establish some basic relationships. Based on the results of the present approach, a general relationship is obtained between the processing time and energy: the processing time is inversely proportional to the utilized energy in the specific process. That is an inherent property of material.
\end{abstract}

Keywords: Electron-photon interaction, energy density, optical pulse length, processing time, time-energy relationship.

\section{INTRODUCTION}

Processing time is an important, characteristic property of every process in physics. Therefore, a question can be arisen: is the processing time an inherent property of the material? In this paper that very generic question is investigated in details. For that purpose the electron-photon interaction is used as a good example.

The electron-photon interactions have always been very interesting phenomena. They have already been discussed in many excellent papers. So far the best explanations come from quantum physics. Spontaneous emission can be calculated very accurately using quantum electrodynamics $[1,2]$. This way, statistical results are obtained, because the process is taken statistical. By other words, it is possible to calculate probabilities of large numbers of interactions.

The aim of this paper is to find understanding and explanations for electron-photon interactions based on a simple new approach. The transition of an electron from an upper energy level to a lower level and the accompanying photon emission can be assumed as an instantaneous process. That is utilized in this paper because currently a single photon emission is already feasible for performing experiments [3].

The main goal of the investigation is to establish some basic relationships for the process of photon generation. Its properties can be described more easily if we consider the generation of photons in a semiconductor material. In this paper that method is used for the investigations which are based on the present new approach to obtain some more results for the properties of photon generation. The derived relationships are in good conformity with the well-known properties of photon generation, however, they provide some new results as well.

\section{PHOTON GENERATION}

First, we consider the photon generation in a direct band gap semiconductor material based on its well-known process

*Address correspondence to this author at the Budapest University of Technology and Economics, Hungary; E-mail: tibor.berceli@mht.bme.hu
$[4,5]$. According to that when an electron is in the conductance band, it is at a stable energy level, consequently there is no radiation. When the electron transits from the conductance band to the valence band a photon can be emitted. Then, when the electron is in the valence band it is again at a stable energy level, there is no radiation. Accordingly, radiation can occur during the time interval, when the electron gets from the conductance band to the valence band. The time interval of this transition is equal to the processing time of photon generation.

Now the question arises: can the transition time be an immediate energy change without taking any time, or does it take some time although a very short time? We can get the answer to this question by considering that the generated photon has a specific frequency. On that basis we propose an approach to estimate the time interval of electron transition which is equal to the processing time of photon generation.

In a direct band gap semiconductor material, there is an energy difference between the conductance band and the valence band, called band gap energy. The energy of the radiated photon is equal to the band gap energy. The frequency, $f_{p h}$ of the photon is given by the well-known equation $[6,7]$ :

$f_{p h}=\frac{E_{b}}{h}$

where $E_{b}$ is the band gap energy, and $h$ is the Planck constant.

When we try to estimate the processing time of photon generation we can use Eq. (1) as a basis. This equation tells us that the photon has a specific frequency and this frequency is dependent only on the band gap energy $[5,6]$.

We consider the photon as the smallest amount of radiated energy at a specific frequency. Accepting this concept there are still some questions which can be arisen. One of these questions is: what is the processing time of photon generation?

\section{PROCESSING TIME}

The question is the following: is the radiating transition of the electron an immediate process in time or does it need 
some time? In the first case the transition process could be described by a Dirac delta function in time [8]. That would mean that the generated photon would not have a specific frequency which is not in agreement with the experimental results or by other words it is not a realistic approach. Therefore the second assumption is more likely, i.e. the transition of the electron from the conductance band to the valence band takes some time, although this time is extremely short. That extremely short transition time is the duration of photon generation or by other words the processing time of photon generation.

How can we estimate the processing time? A photon can be represented by an extremely short electromagnetic pulse which has the duration equal to the generation time of the photon. That pulse has to be in connection with the photon frequency. That means the fundamental frequency of the Fourier series representing the electromagnetic pulse has to be equal to the photon frequency. Based on that requirement a possible assumption is to take a single, one period of a raised cosine function representing the electric component of the pulse with the duration equal to the period time of the photon frequency. This way the processing time of photon generation is:

$\tau_{p h}=T_{p h}$

Here $\tau_{p h}$ is the processing time of photon generation and $T_{p h}$ is the period time of photon frequency: $T_{p h}=1 / f_{p h}$. In that case the energy is concentrated into the period of the photon frequency. In the following investigations that assumption is used.

The next question is as follows: is the generation time constant or is it dependent on some effect? The answer is: the generation time is not constant, it is dependent on the band gap energy. From Eqs. (1) and (2) we get:

$\tau_{p h}=\frac{h}{E_{b}}$

If the band gap energy is higher the generation time is shorter, or by other words the radiated pulse or burst is shorter.

\section{UNCERTAINTY PRINCIPLE}

We use now the uncertainty principle for estimating the processing time in a different way. Based on Eq. (1) there is a relation between the period time $T_{p h}$ of the photon frequency and the band gap energy $E_{b}$ generating the photon:

$T_{p h}=\frac{h}{E_{b}}$

When we want to determine the processing time we have to consider the Heisenberg uncertainty principle $[9,10]$. Accordingly, we get:

$\Delta t \Delta E \geq \frac{h}{4 \pi E_{b}}$

Here $\Delta t$ is the change in time due to the $\Delta E$ change in the energy and $h$ is the Planck constant. In our case:

$\Delta E=E_{c}-E_{v}=E_{b}$
$E_{c}$ is the energy level of the electron in the conductance band and $E_{v}$ is the energy level of the electron in the valence band. Their difference is the band gap energy $E_{b}$.

Further on, $\Delta t$ is the time elapsed from the start of the process up to the end of the process, or by other words $\Delta t$ is the processing time, i.e. the duration of photon generation:

$\Delta t=\tau_{p h}$

Based on Eqs. (3), (5), (6) and (7) we can write:

$\tau_{p h}=\frac{h}{E_{b}} \geq \frac{h}{4 \pi E_{b}}$

Consequently, the estimation for the duration of photon generation is in conformity with the Heisenberg uncertainty principle [9-11].

\section{PROCESSING TIME AND ENERGY RELATIONSHIP}

We presented an approach to estimate the duration of photon generation. Although we consider the photon having a specific generation time and energy it cannot be separated into smaller parts because the photon has been generated by a single or unique energy transition of an electron in the atom.

As the duration of photon generation is inversely proportional to the photon frequency and its frequency is directly proportional to the band gap energy - see Eq. (1) we can state: the generation time is inversely proportional to the band gap energy.

Furthermore, it can be stated for a general case: the processing time is inversely proportional to the energy utilized for performing a specific process. Here it is assumed that the other parameters of the process e.g. electric charge, mass, temperature, material, etc. are unchanged.

The main goal of the investigation on the duration of photon generation is not to determine its exact value but to establish some basic relationships. Evaluating the results of the present approach we can formulate some relationships:

- $\quad$ the transition of an electron from one energy level to another one takes some time,

- the duration of photon generation is determined by the transition time of the electron emitting the photon,

- the duration of photon generation is estimated to be equal to the period time of its frequency,

- the duration of photon generation is inversely proportional to its frequency,

- $\quad$ the processing time or duration of photon generation is inversely proportional to the band gap energy.

Based on the previous statements a more general rule can be suggested: the processing time is inversely proportional to the energy involved in the process. Furthermore we can state: time is an inherent property of processes in nature.

\section{LENGTH OF OPTICAL PULSES}

The generation of an optical pulse is a crucial task for many applications. There is a trend to generate shorter and shorter pulses. The question arises: is there a lower limitation on the pulse length. The answer is: yes. Determining that 
limitation we can assume that all photons of the optical pulse are generated exactly at the same time which would give the possible shortest optical pulse. As it has already been assumed the process of photon generation can be described by a single, one period long raised cosine function. In that case the width of that pulse between the $-6 \mathrm{~dB}$ power points is equal to the half processing time. Therefore, we can take that value as the lower limit $\left(\tau_{o p}\right)$ for the optical pulse generation at a specific frequency:

$$
\tau_{o p} \geq \frac{\tau_{p h}}{2}=\frac{h}{2 E_{b}}
$$

That idealistic situation cannot be realized therefore it can be stated: it is not feasible to generate optical pulses shorter than the half of the photon generation time. Consequently if we want to generate shorter optical pulses then it can only be achieved at a higher frequency or by other words at a shorter wavelength.

That is in good agreement with the best published results. The shortest optical pulse which was experimentally achieved at the wavelength of $750 \mathrm{~nm}$ was $5.5 \mathrm{fs}$ (femtosecond) [12]. At this wavelength the limitation based on Eq. (9) is 1.25 fs. Another extremely wonderful experimental result provided an 80 as (attosecond) optical pulse in the $\mathrm{x}$-ray wavelength band [13]. The limitation given by Eq. (9) is about 16.7 as if the longest wavelength $(10 \mathrm{~nm})$ of the $\mathrm{x}$-ray band is used for determining the limitation. At shorter wavelength the limit for the generation of a pulse is much smaller.

\section{ENERGY DENSITY}

Based on Eq. (1) the energy of the photon is proportional to the band gap energy. Therefore, a higher frequency photon carries higher energy. However, the question arises: what is the energy density of photon generation?

The energy density of a process $(\rho)$ can be defined as the ratio of the energy involved in the process $(\Delta E)$ and the processing time $(\Delta t)$ :

$\rho=\frac{\Delta E}{\Delta t}$

Therefore, in the case of photon generation the energy density is:

$\rho_{p h}=\frac{E_{b}}{\tau_{p h}}=\frac{E_{b}^{2}}{h}$

The energy density of photon generation is proportional to the square of the band gap energy.

By considering the relation between the generation time and the band gap energy it can be concluded: when the band gap energy is higher the duration of photon generation is shorter, therefore the energy density is even higher. By other words the energy density is higher at higher frequencies due to the higher band gap energy and also due to the shorter generation time. This way the ratio of the photon energy densities at different frequencies is proportional to the square of the ratio of their band gap energies (or their frequencies): $\frac{\rho_{p h}\left(f_{2}\right)}{\rho_{p h}\left(f_{1}\right)}=\left(\frac{E_{b 2}}{E_{b 1}}\right)^{2}=\left(\frac{f_{2}}{f_{1}}\right)^{2}$

The indices 1 and 2 refer to quantities at two different photon frequencies.

This quadratic equation holds when photons are generated in a direct band gap semiconductor material. That statement does not depend on the estimated value of the generation time because the same approach is used at every photon frequency. It is worth mentioning that similar results have been obtained by Planck for black body radiation [5] showing not a linear but a faster increase in the spectral energy density with enhancing frequency of radiation.

Considering the interaction of photons and material or by other words the effect of electromagnetic radiation on material, it can be stated: this effect is dependent not only on the quantum energy but also on its density. That means this effect is not dependent only on the photon frequency but also on the square of the photon frequency. This relationship has significant consequences in biology as well: the effect of electromagnetic radiation on biological tissues is dependent not only on the total energy of a photon but also on its density: a higher density results in a stronger effect.

\section{PASSING TIME}

Concerning the nature of time several questions can arise. Whether time is a general property of nature or is it an artificial parameter introduced by us to describe processes in nature? Is it a property of material or is it only a parameter introduced by us? And, what is the meaning of the phrase "passing time" or "time is passing"? Is time always passing with the same speed? Can our measure of time, e.g. the atomic clock be considered as an inherent property of nature? These questions are very important, therefore how can we get a right answer to them.

We can state: time is passing as changes occur in the state of the material, like changes in its energy level, location, motion, composition, etc. To perform such a change, some energy is needed. If the inherent energy of a specific physical process which is utilized to carry out the change in the state of material is higher, the change is carried out in a shorter time.

We also can say: processing time is the consequence of the utilized (or delivered) energy in a process changing the state of the material. Naturally, the utilization (or delivery) of energy is dependent on external conditions as well, therefore, the same conditions are assumed when the relationship between time and energy is considered.

As another consequence of this relationship we can state: due to the quantized property of energy, time is also quantized. Therefore, a quantum of time belongs to a quantum of energy or by other words the quantum of time is dependent on the quantum of energy involved in a process in nature.

The relationship between energy and time can be extended to many other physical processes. Further on, it can be seen: time is a phenomenon inherent in physical processes because there is a direct connection between time and energy 
involved in a specific physical process. This relationship presents connection between energy and time.

Based on the afore mentioned relationship between time and energy it can be concluded: getting energy from fossil fuels by burning them the duration of the process is not short because the delivered energy from a unit amount of the material is relatively not high. However, getting energy in a nuclear plant from a radioactive material is a much faster process because the delivered energy from a unit amount of the material is rather high. Furthermore, getting energy in a fusion reactor is an extremely fast process because the delivered energy from a unit amount of the material is enormously high. Due to the very fast delivery of a high amount of energy, the control of the fusion process is very cumbersome.

The relationship between energy and time is now applied for a material having relativistic speed, or by other words for a material moving with a velocity approaching the speed of light. In that case the mass of the material is increased and therefore the utilization or delivery (emission) of the same amount of energy will occur during a longer interval of time. By other words at relativistic speed, time is slowed down, the same action needs longer time. That is in complete agreement with the relativity theory [7].

\section{MEASUREMENT OF TIME}

Now the question arises: how we can measure the duration of a process or by other words how can we determine the time as it is passing? We use again the definition: time is passing as changes in the state of material occur (including its energy level). Therefore, we utilize some kind of energy oscillation for that purpose. Earlier a pendulum was applied to measure how time was passing. In that case periodic static and dynamic energy changes occurred. Presently, a quartz crystal oscillator is used which means periodic mechanical and electrical energy changes. The oscillation has to be stable which means the frequency of oscillation has to be constant. Then we take a counter and as counting proceeds time is passing. According to that method time is passing always with the same speed because the energy fluctuation in the oscillation process is kept constant. However, this clock can only be used as a reference on our globe. Can we generalize that process for the universe?

As it has been stated, time is passing with such a speed as changes in the state of material are observed. E.g. on a cold planet far from any energy source nothing happens at all. We can say time is stopped there. However, close to the sun a huge amount of energy is encountered and changes in the state of material take place very frequently, i.e. in a very short time. How could our clock work there?

\section{CONCLUSIONS}

In this paper a new simple approach is presented which gives results on the duration of photon generation, its energy density and on the relationship between the processing time of photon generation and the utilized energy. That relationship can be extended to other processes stating as a general rule that the processing time is inversely proportional to the energy involved in a specific process.

\section{ACKNOWLEDGMENTS}

The author acknowledges the European Union Network of Excellence projects called ISIS and NEFERTITI as well as the Hungarian National Scientific Research Foundation (OTKA projects: T030148, T026557, T034520) for the continuous support to his research work.

\section{REFERENCES}

[1] Yariv A. Quantum Electronics. $3^{\text {rd }}$ ed. New York: John Wiley; 1989.

[2] Bohr N. In: Wheeler JA, Zurek WH, Eds. Quantum Theory and Measurement. Princeton: Princeton University Press 1984; pp. 949.

[3] Jacques V, Wu E, Grosshans F, et al. Experimental realization of Wheeler's delayed-choice gedanken experiment. Science 2007; 315: 966-8.

[4] Saleh BEA, Teich MC. Fundamentals of Photonics. New York: John Wiley 1991.

[5] Sze SM. Physics of Semiconductor Devices. 2nd ed. NewYork: John Wiley 1981.

[6] Einstein A. Zur Quantentheorie der Strahlung (On the Quantum Theory of Radiation). Physikalische Zeitschrift 1917; 18: 121-8.

[7] Stachel J. Ed. The collected papers of Albert Einstein. Princeton: Princeton University Press 1989.

[8] Dirac PAM. Quantum Mechanics. London: Oxford University at the Clarendon Press 1970

[9] Heisenberg W. The Physical Principles of Quantum Theory. London: Dover Publication 1930.

[10] Bohr N. The quantum postulate and the recent development of atomic theory. Nature (Suppl) 1928; 121: 580-90.

[11] Burkhardt ChE, Leventhal JJ. Topics in Atomic Physics. New York: Springer 2006.

[12] Uiberacker M. Pervak V, Apolonski A, et al. Attosecond real-time observation of electron tunnelling in atoms. Nature 2007; 446: 62732.

[13] Krausz F. Ivanov M. Attosecond physics. Rev Modern Phys 2009; 81: $163-234$ 